\title{
An RFID-Enabled Automated Warehousing System
}

\author{
Qian Wang, Saleh Alyahya, Nick Bennett, and Hom Dhakal
}

\begin{abstract}
It has been seen a rising trend in recent years for online shopping businesses across the UK. Customers prefer to place their orders online to purchase goods and ordered goods are dispatched directly from regional warehouses (or distribution centres) to customers' door steps. A number of studies through a literature review indicated that future generation warehouses may be designed and implemented as more centralised distribution centres as this is the most cost-effective way to run businesses of this form for many larger manufacturers, suppliers or retailers. These companies have therefore been seeking for even more efficient and effective methods for storing, picking and dispatching goods in increasingly centralised distribution centres in which a novel design of warehousing systems is desired. This paper presents a framework of an RFID-based management system as part of a proposed future generation warehouse design through application and integration of fast-growing IT technologies (radio frequency identification (RFID) tags, wireless sensors and communication networks) to maximise the warehouse capacity and achieve a better visualisation and real-time visibility of inventory control and data handling management.
\end{abstract}

Index Terms-Warehouses, inventory, RFID, automation.

\section{INTRODUCTION}

In the past decade, there has been an exponential increase in the number of online shopping customers across many countries including the UK. These customers prefer to order goods online and expect a fast delivery of ordered goods to be dispatched directly to their door steps. Because of this type of shopping habit, many traditional stores or warehouses are no longer suitable for satisfying the demand of online shopping customers. A literature study showed that future generation warehouses may be designed and implemented as more centralised distribution centres that partly replace traditional stores of manufacturers, suppliers and retailers in the UK. This requires a novel design of cost-effective storage and retrieval systems as a key element of distribution centres for storing, picking and dispatching goods. Implementation and integration of fast-growing IT technologies has also demonstrated great improvement opportunities of a warehouse in terms of tighter inventory control, shorter response time and greater variety of SKUs (stock keeping units). These IT capabilities can be enhanced by using smart-labels such as radio frequency identification (RFID) tags (to replace traditional barcode-labels) and automatic identification (Auto-ID) sensors, together with cable and/or wireless communication networks and indoor warehouse management systems (iWMS) [1].

In recent years, application and implementation of

Manuscript received January 5, 2015; revised April 24, 2015.

The authors are with the School of Engineering, University of Portsmouth, Portsmouth, PO1 3DD, UK (e-mail: qian.wang@port.ac.uk).
RFID-related techniques has been increasingly becoming popular particularly in logistics and supply chain sectors. In a latest literature review by Lim et al., it discussed the previous and current status and a future trend in RFID-based applications, benefits and obstacles [2]. In terms of research and development in the relevance to this study, Wang et al. proposed a conceptual design of future generation automated warehousing systems by incorporating fast-growing IT technologies such as RFID tags, wireless sensors and communication networks into a modular mechanism of automated storage and retrieval systems, which have a feature of scalability and configurability [1]. Wang et al. developed a digitalised warehouse management system (DWMS) by applying RFID techniques into a tobacco company. Within such an RFID system, a set of basic events with storage and retrieval policies are defined as event-condition-action (ECA) rules to improve the feasibility and flexibility of the DWMS [3]. Chow et al. examined an RFID-based resource management system (RFID-RMS) used for logistics to enhance efficiency and productivity of warehouse operations by tracking and optimizing utilisation of resources. In order to achieve this, a customised route-optimizing model was developed by reading real-time data directly from RFID tags to determine an order of picking operations performed by material handling equipment. A pure-integral-linear programming model was developed based on a branch-bound algorithm to determine an optimum travel distance of a forklift [4]. Liu et al. studied on an RFID system used for monitoring operations in positioning and loading material handling facilities in a warehouse. The experimental result also showed an improvement in terms of rack space utilisation and reduction in work-related operational errors [5]. Poon et al. introduced an RFID-based logistics resource management system by facilitating a collection of sharing data in a warehouse database for managing order-picking operations [6]. Ting et al. applied an RFID-based inventory control and management system (RICMS) into a manufacturing enterprise in order to enhance the product life cycle management (PLM) system [7]. Ross et al. presented a product-tagging and processing decision maker based on major functions (receiving, storing, picking and shipping) occurring within a typical warehouse. This work provided an opportunity to develop a simulation model that can be used for evaluating operations of a distribution centre and examining the impact on alternative RFID implementation strategies [8].

In other related studies, Sahin et al. examined an issue of data inaccuracy of warehouse inventory and explored a way in which this issue may be tackled using an RFID system focusing on parameters that may affect the inaccuracy on product availability, supply chain performances, appropriate inventory counting policies and adjustability of safety stocks and replenishment policies [9]. Cakici investigated 
incremental benefits of using RFID tags over barcodes for pharmaceutical inventory management [10]. Xu et al. introduced a warehouse management optimization method based on varying SKU (storage keeping unit) levels by developing an RFID-enabled warehouse [11].

Implementation of RFID systems through integration is an important aspect that needs to be addressed for the warehousing system. Liu et al. conducted a case study by integrating an RFID system into an enterprise resource planning (ERP) system using the Oracle application implementation methodology (AIM). The developed system included two modules: an electronic receiving module and an inventory transaction module [12]. Xiaoguang et al. proposed an adaptive communication framework of a warehouse management using RFIDs and wireless sensor networks (WSN). The proposed system used a reliable protocol for a development of network architecture. By integrating the RFID and WSN technologies, it offered an opportunity to have a reliable identification and tracking function of goods [13]. Jehng et al. investigated an automatic conveyor system on which material flow was monitored and traced in a real time manner using RFID tags attached with products. The collected data were saved in a database constructed by the Laboratory Virtual Instrumentation Engineering Workbench (LabVIEW) software [14]. Yoo et al. focused on development of a middleware platform in application of an RFID system for reverse logistics. The work proposed a network infrastructure that supports logistic activities from the recycle to the disposal of product materials by adding reverse logistics to the existing forward logistics [15]. In brief, it has been widely accepted that applications of RFID techniques create a better visualisation and traceability of inventory data therefore facilitating automation of storage and retrieval assignments in a warehouse. Compared to a conventional warehouse using the barcode approach, implementation of RFID systems has also demonstrated a significant improvement in warehouse data handling efficiency and space utilisation [16].

A feasibility study by Wang et al. indicated that an RFID-enabled automated warehousing system has several advantages over the conventional automated warehouses. Within such an automated storage and retrieval mechanism, each item in a tote (or a tote containing identical items) is attached with an RFID tag so that these in-store items can be traced, sorted and inventoried in a real-time manner through an integrated RFID-inventory management system that interacts with the mechanism of automated storage and retrieval systems. By implementing such an RFID-enabled warehousing system, each item can also be stacked onto storage racks $(\mathrm{S} / \mathrm{R})$ at a random location wherever a place is available for incoming goods. Thus, an item can also be distributed from an $S / R$ at a random location throughout the warehousing system. Using this material-handling method it can significantly improve efficiency of warehousing operations and utilisation of warehousing space.

This paper presents a continuous study in developing an RFID-inventory management system as part of future generation automated warehousing systems proposed by Wang et al. [1]. The research work involves a development of an RFID-inventory management system that has capability to interact with a mechanical control system embedded in an automated storage and retrieval system. In theory, this proposed RFID-enabled automated warehousing system can also be incorporated into centralised distribution centres through supply chain networks leading to a better accuracy of real-time inventory data management and a more even distribution of incoming and outgoing goods in local regions to accommodate varying customers' demands on a daily basis.

\section{The RFID-EnABLED AutOMATED StORAGE AND RETRIEVAL SYSTEM}

The automated storage and retrieval system (AS/RS) is a material handling mechanism which is a key element of automated warehouses or distribution centres. Fig. 1 illustrates the RFID-enabled automated storage and retrieval racks (AS/RR) as core component of the proposed warehousing system. The module is designed as a standardized element for manufacturing and assembly, although each module can be of different sizes and arrays in a module can be configured easily in many different ways, i.e., capacity of a warehouse is adjustable. In the warehouse, items are pre-loaded onto pallets named totes; totes need not be of identical sizes. Each item in a tote (or a tote containing identical items) is attached with an RFID tag and each item in a tote is thereafter tracked and manipulated by the developed RFID-inventory management system throughout the warehouse.

A typical RFID reader is a microcontroller-based radio transceiver that powers an RFID tag using the time-varying electro-magnetic fields (EMF) generated from an RFID antenna. Once an RFID tag is powered, it can receive commands from an RFID reader. An RFID tag is composed of two essential components: an antenna and a computer chip. The computer chip is used to store data while the antenna allows data communication between an RFID tag and an RFID reader through a wireless signal transmission. Each RFID tag has a unique identification (UID), which can be used to uniquely identify an RFID tagged item. The collected RFID information data by an RFID reader can be transferred to a host PC as database for data processing and storage. Thus, the hardware of the proposed RFID-based warehouse management system consists of the following key elements:

1) RFID Readers

2) RFID tags

3) A host computer

Two types of RFID tags (active and passive RFID tags) can be used depending on a range of RFID reading performance at a location of an AS/RR. By using RFID tags in the proposed automated warehouse, SKUs can be distributed evenly and dispatched randomly at varying locations wherever a place is available for incoming and outgoing goods. This design significantly facilities operations of storage, retrieval and replenishment in the warehouse.

The module comprises two types of powered conveyors aligned next to one another as illustrated in Fig. 1; these are input conveyors (storage racks) and output conveyors. The entire operation of each conveyor system is controlled by a 
PLC (programmable logic controller) that communicates with mounted sensors via a local area network (LAN). Within the RFID-inventory management system, a chosen SKU can be released by the system based on a number of assignment policies or rules. These rules include for example the rule of "being nearest to a collection point and/or a modular arm which is free or adjacent to the chosen SKU" and so on, which is also explained in other section below. Overall, this design significantly increases warehouse capability, flexibility and responsiveness for storing, picking and dispatching an item through the storage and retrieval system.

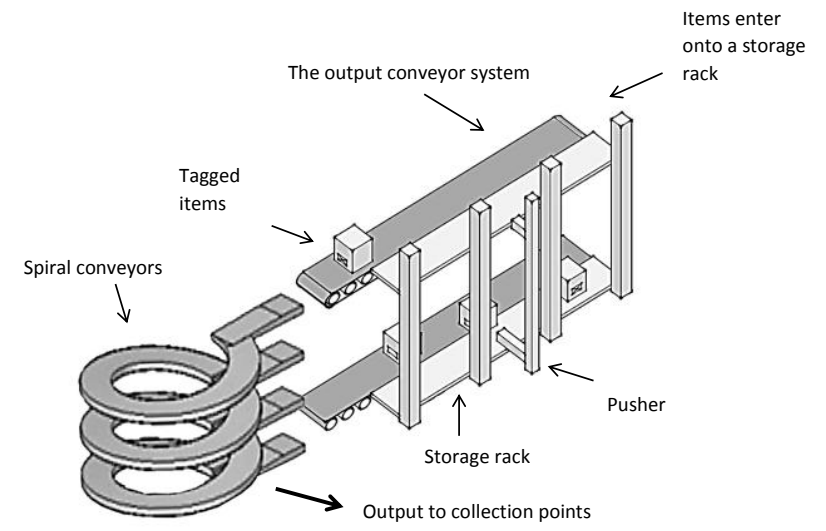

Fig. 1. Structure of the automated storage and retrieval system.

\section{MEASUREMENT OF THE StORAGE AND RETRIEVAL SYSTEM}

Fig. 2 also illustrates the key components and corresponding geometric parameters of the proposed storage and retrieval system illustrated in Fig. 1: (1) output conveyor; (2) storage rack; (3) height of a storage rack $(H)$; (4) length of a storage rack $(L) ;(5)$ depth of a storage rack $(D) ;(6)$ a pusher; (7) length between an output conveyor and an spiral conveyor at a level (Lsc); (8) entrance to a spiral conveyor; (9) length of a single spiral conveyor (Lss); (10) a spiral conveyor; (11) length of a spiral conveyor to a collection point $(L s p)$. The aim of the following work is to determine a total travel time an item needs from the moment when a pusher is activated to push a selected item in a tote onto an output conveyor to the moment this item travels down from a spiral conveyor to a collection point. Fig. 3 shows a two dimensional cross-section diagram based on Fig. 1-2. The pusher $(P n)$ can move simultaneously in both horizontal and vertical directions from one location $(i, j)$ at where the pusher currently stays to another location $(m x, n x)$ at where an identified item is selected to be pushed onto the output conveyor in the AS/RR illustrated in Fig. 1. The following assumptions are used in this case study.

1) The pusher is capable of moving instantly to obtain a stable and constant speed without an acceleration/deceleration.

2) Because the pusher is capable of moving simultaneously in both vertical and horizontal directions at a stable and constant speed, this implies that the pusher can move along the linear route $L d$ to a specified location before pushing a selected item from a storage rack onto an output conveyor.

3) Each item in a tote is pushed onto an output conveyor instantly without any delay.

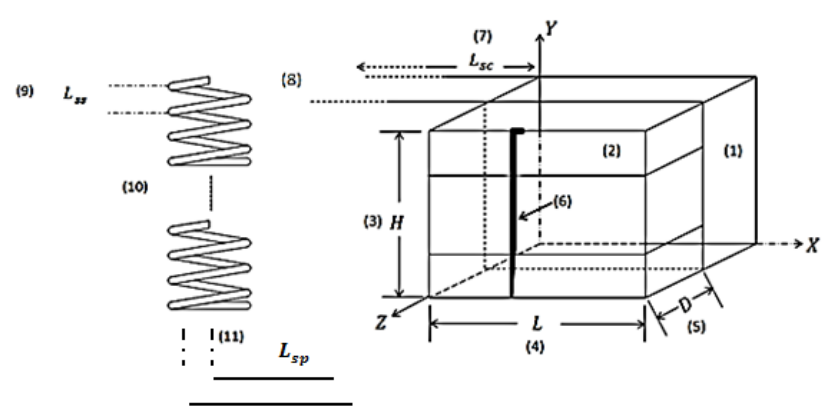

Fig. 2. Geometric parameters corresponding to the key components of the storage and retrieval system.

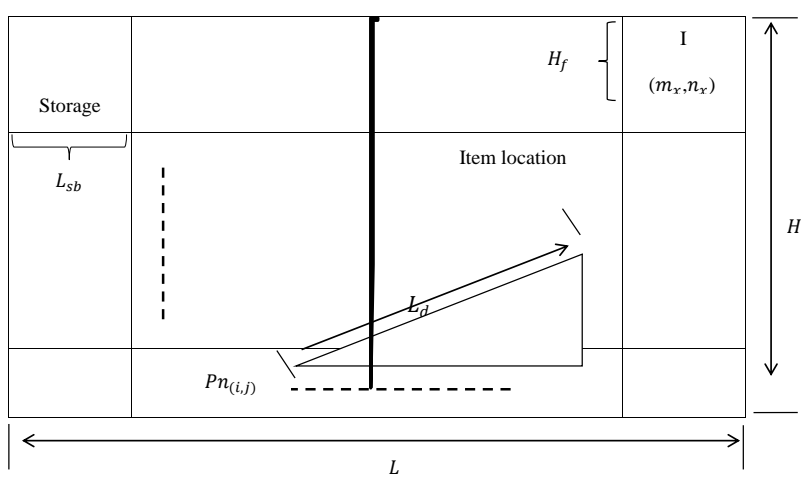

Fig. 3. Geometric parameters in a storage rack.

The following notations are used in this study.

- I: an item at a row $m x$ and a column $n x(m x, n x)$ of an AS/RR.

- Pn: a pusher at a location $(i, j)$.

- $L s b$ : length of a storage room of an AS/RR.

- Lsc: length from a position of a selected item in a tote to be pushed onto an output conveyor to a position where this item in a tote travels at the end of an output conveyor.

- Lss: length of a single spiral conveyor.

- Lsp: length from the end of a spiral conveyor to a collection point.

- $L d$ : distance between a pusher and a selected item.

- $V c$ : speed of a conveyor.

- $V p$ : speed of a moving pusher along $L d$.

- Vpp: speed of a moving pusher to push an item onto an output conveyer.

- Vs: speed of a spiral conveyor.

- Tm: time needed for a pusher to move to a selected item.

- Tp: time needed for a pusher to push a selected item onto an output conveyor from an input conveyor (i.e., a storage rack).

- Ts: travel time of a selected item in a tote along an output conveyor to a spiral conveyor.

- Tsc: travel time for a selected item in a tote from the top level to the bottom level of a spiral conveyor.

- Tse: travel time from the end of a spiral convey to a collection point.

- Ttt: time needed to pick up a selected item.

- Tci: Total travel time of a selected item throughout the storage and retrieval system to a collection point. 
Illustrated in Fig. 3, assuming an item has a location ( $m x$, $n x)$ and a pusher has a random location $(i, j)$ in a storage rack. Thus, the distance $L d$ between the activated pusher and a selected item is given by:

$$
L_{d}=\sqrt{\left(m_{x}-i\right)^{2}+\left(n_{x}-j\right)^{2}}
$$

Therefore, the travel time $T m$ is given by:

$$
T_{m}=\frac{L_{d}}{V_{P}}
$$

where, $V p$ is a speed of the moving-pusher along $L d$. We define $T p$ is a travel time from the moment the pusher starts to push a selected item to the moment that the selected item has been pushed onto an output conveyor, it is given by:

$$
T_{p}=\frac{D}{V_{P P}}
$$

where, $D$ is a depth of the storage rack in which the selected item is located. Vpp is a constant speed of the moving-pusher to push the selected item onto an output conveyer. Knowing Lsc refers to a distance from the centre of a tote containing the selected item to the centre of the tote at the end of the output conveyor. $L s b$ is the length of a storage room containing each item in a tote, $n x$ refers to the location $x$ in row $n, V c$ is a constant speed of the output conveyor. Thus, the total travel time of the selected item $T s$ is given below:

$$
T_{s}=\frac{L_{s b} \times\left(n_{x}-0.5\right)+L_{s c}}{V_{c}}
$$

The item travels down through a powered spiral output conveyor from the top level to the bottom level towards a collection point where the item is collected for packaging. Thus, the possibly required travel time $T s c$ is calculated by:

$$
T_{s c}=\frac{m_{x} \times L_{s s}}{V_{S}}
$$

where, Lss is the length of a single level spiral conveyor. $m x$ refers the location of the horizontal level $x$ in row $m$. Assuming that Tse is a travel time that the item needs from the end of a spiral conveyor and a collection point. Lsp is a travel distance between the end of a spiral conveyor and a collection point. $V c$ is a constant speed of the conveyor.

$$
T_{s e}=\frac{L_{S P}}{V_{c}}
$$

Hence, the total travel time $T t t$ for the selected item is obtained by:

$$
T_{t t}=\sum_{n=1}^{n} T_{m_{-} c i}+T_{s_{-} c i}+T_{s c_{-} c i}+\left(T_{p}+T_{s e}\right)
$$

In order to choose a selected item that needs a least travel time identified by the RFID-inventory management system throughout the storage and retrieval system, the minimal travel time $T c i$ can be obtained by:

$$
T_{c i}=\min \left[T_{t 1}, T_{t 2}, \ldots \ldots \ldots \ldots \ldots, T_{t t}\right]
$$

\section{The WAREhousing MANAGEMENT SyStem}

Fig. 4 shows a simplified RFID inventory management system for the proposed automatic warehousing system in a distribution centre at where an RFID reader is mounted on the portal gate and it takes a reading of RFID-tagged incoming and outgoing goods each time when these items enter into or exit from the distribution centre. Each pusher mounted on an AS/RR also contains an RFID reader that communicates with a local controller, which transmits collected data via a middleware to a central warehousing database. The middleware is the software translation layer between an RFID reader and an enterprise system. Once an in-store item is ordered by a customer online, the RFID-inventory management system is notified after receiving the order and it checks availability from the warehousing database, which contains real-time information collected by RFID readers via an indoor wireless local area network (iWLAN) through the controller. The collected information data include a unique identity and a description of each ordered item as well as an SKU number of the warehouse. Once an ordered item is identified by the RFID-inventory management system, a pusher is activated by a PLC (programmable logic controller) to push the selected item in a tote onto an output conveyor. The item will then be transported by the output conveyor and it travels along a guided route to a specified destination (collection point) for packaging. The warehouse RFID-inventory database will then be updated as soon as this ordered item is shifted out of the distribution centre through the gate equipped by the RFID-reader.

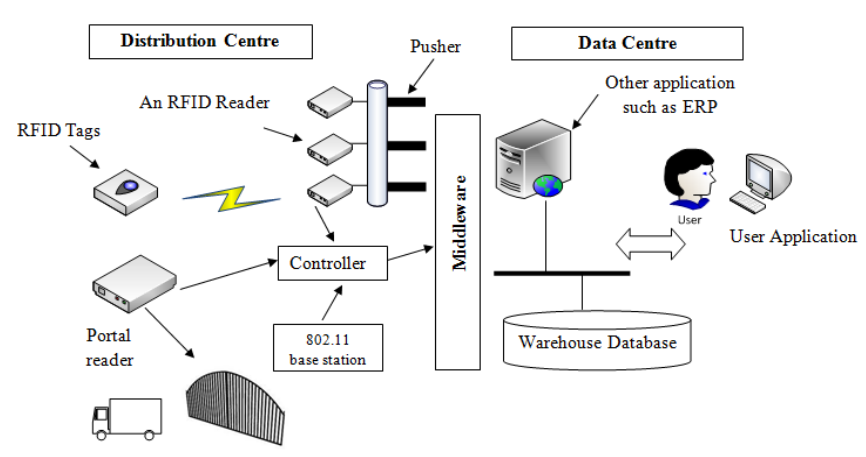

Fig. 4. The RFID inventory management system.

Automated material-handling activities of a pusher are determined by a mechanical control system that generates a demand by executing pre-defined assignment policies, which are a set of selection rules. These rules include such as availability of the selected item, its location closest to a collection point, a shortest route for the selected item traveling to a specified collection point, expiry dates of 
products if applicable and so on. If an item is selected from a group of the same type of items stored in multiple locations of the warehouse, the system will issue a priority based on the pre-defined selection rules to be given to the selected item to initialise a demand to push it onto the output conveyor.

Job scheduling plays an important role in efficiency and productivity of warehousing operations. As stated previously, the proposed RFID-enabled warehousing system allows an item to be stored randomly at varying locations wherever a storage place is available, i.e., the same type of items may have multiple locations. Hence, the developed RFID-inventory management system has capability of identifying a dispatched item by issuing a priority to the selected item based on assignment policies as described above. In order to schedule a job priority for the selected item to be dispatched from the warehousing system, an algorithm was developed to seek an optimal solution for a selected item which has a priority over other items of the same type based on variables in terms of such as expire date and a least travel time to a specified collection point and so on. Under this integrated RFID-enabled warehousing management system, customers place their orders on-line through a web-based platform and the RFID-inventory management system automatically checks its database in terms of availability of each ordered item. As soon as these available items are ordered by a customer after making a payment online, the warehousing system then performs an automatic item-selection process without any human interference.
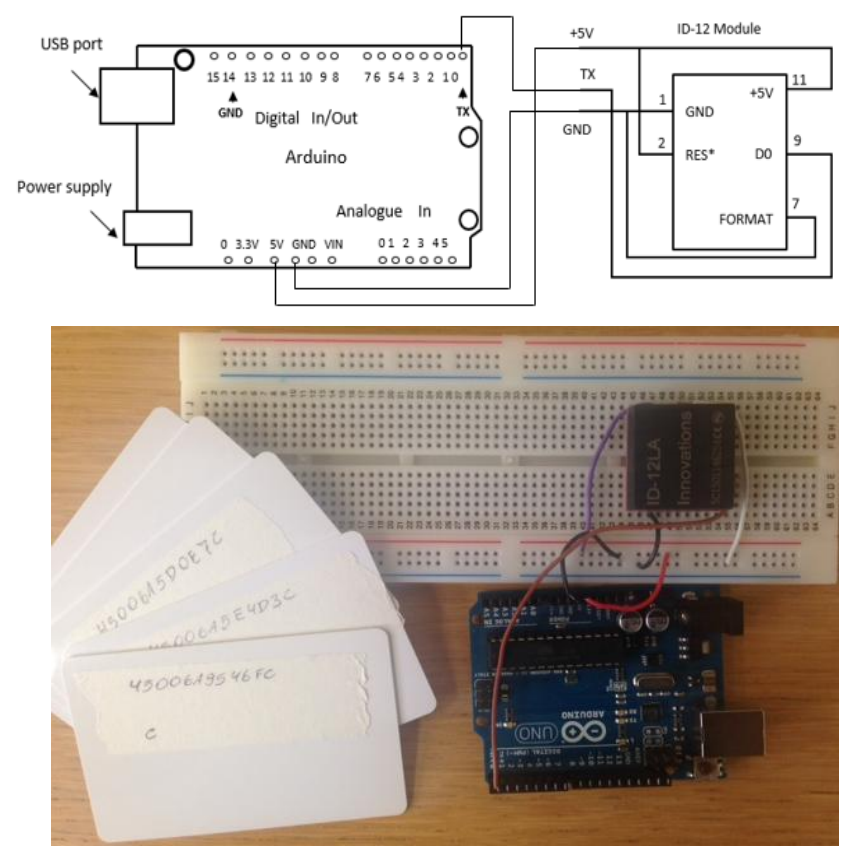

Fig. 5. The Arduino interface.

To carry out a pilot test, a programme was developed using MATLAB based on the developed mathematical model to determine a minimal travel time for a selected item to be dispatched from an AS/RR to a specified collection point. MATLAB allows a matrix manipulation of data plotting function, it can also interface with other programs written in different languages. A prototype of the RFID-inventory management system was built and the backbone of it is an
Arduino interface which interacts with the RFID system as shown in Fig. 5. The ID-12 module was used to perform the task of detecting and reading RFID tags and subsequently printing out RFID codes over an USB port terminal (COM4) as shown in Fig. 6 and Fig. 7 demonstrates part of programming MATLAB codes showing data processing outputs of ordered items based on the developed algorithm. The storage and retrieveal system performs item-picking operations by excuating a set of rules to evaluate conditions of ordered items and carry out relevant actions automatically.

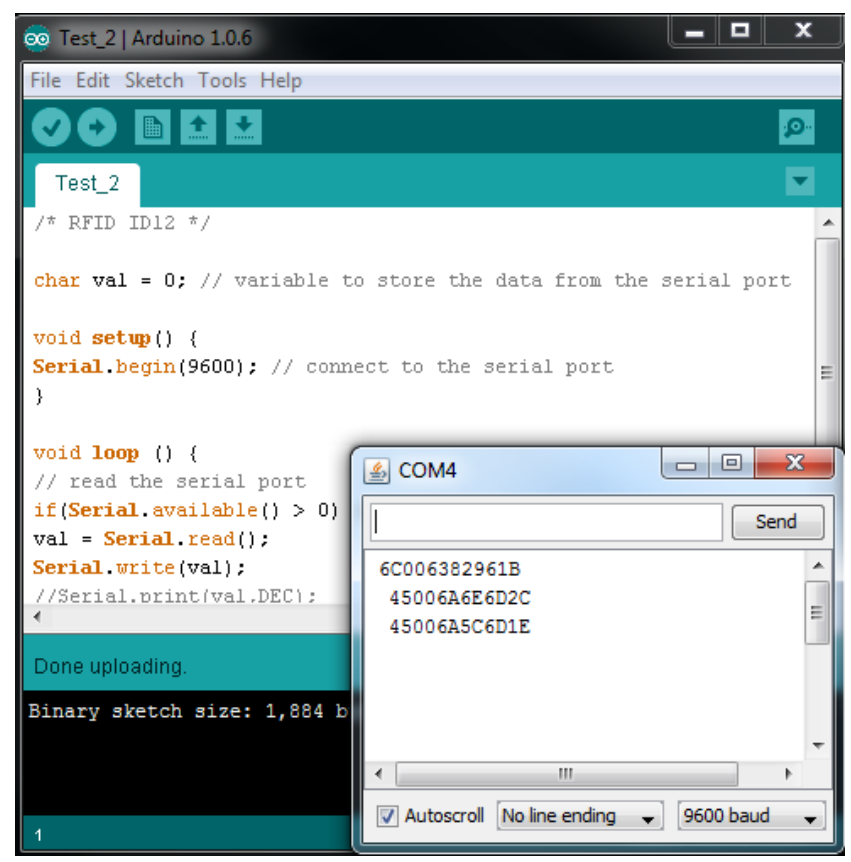

Fig. 6. Arduino reading results

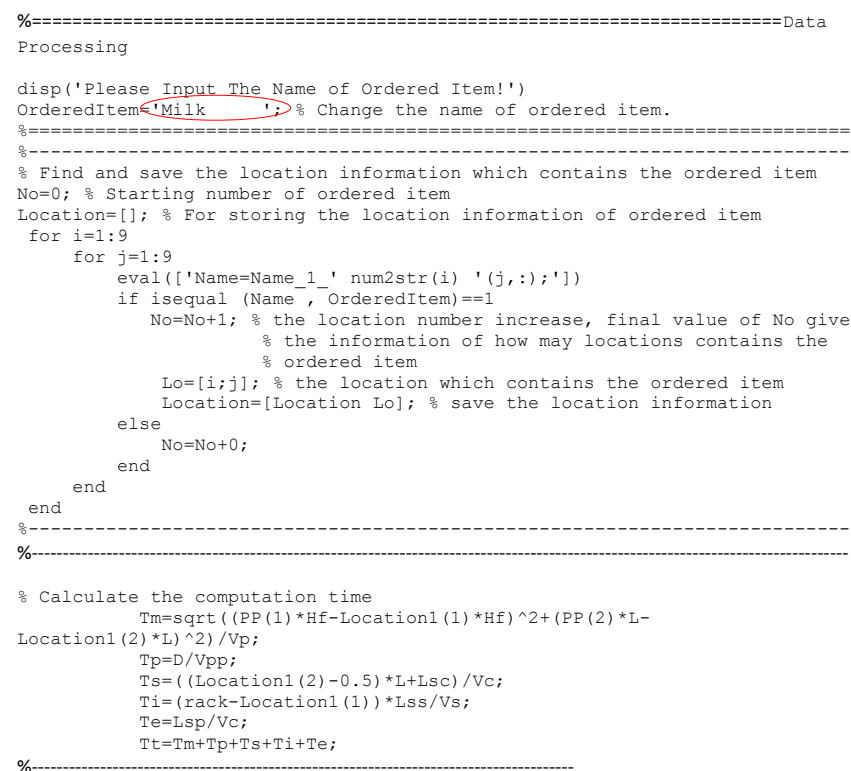

Fig. 7. A screenshot of part of programming codes using MATLAB

Fig. 8 shows an example of information data collected by reading RFID tags in a MATLAB environment based on the following rules:

1) location of an ordered item

2) state of an ordered item

3) identity of an ordered item at a location 
4) A nearest object to a collection point.

The developed RFID-based management system autmatically identifies and detects a number of locations of the ordered item by displaying object rows and columns on storage racks. This implies that seven locations contain the same type of the ordered item. It also shows the name and the current state of the ordered item. Because the ordered item at varying locations has different expiry dates. Based on the developed algorithm, the system will then issue a demand to dispatch the ordered item by assignin a priorty to the selected item based on the closest expiry date and the nearest item to a collection point.
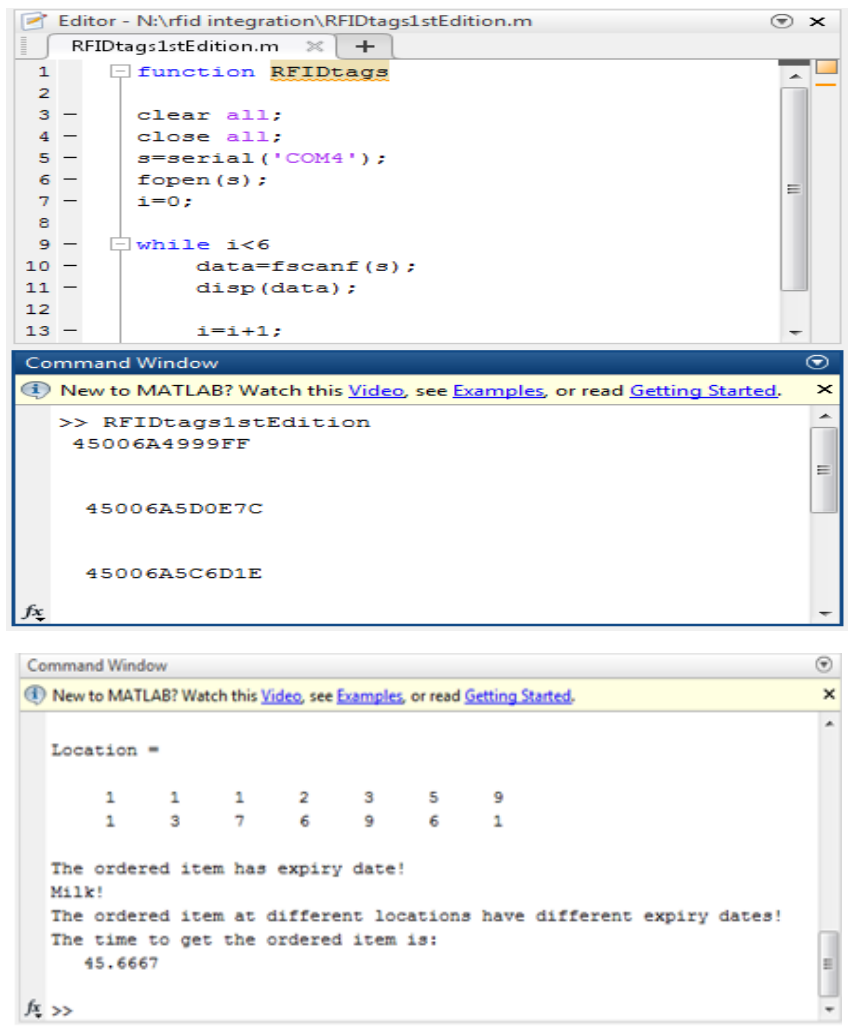

Fig. 8. Result of processed RFID data of an ordered item.

\section{CONClusions}

This paper presents part of a research work aimed at developing an RFID-based warehouse management system in which customers place their orders on-line through a web-based platform; the developed RFID-inventory management system then carries out an availability check on its database for ordered items and then dispatches these items automatically from storage and retrieval racks without any human operations. The feasibility study indicates that the RFID-enabled management system can be incorporated further into the novel design of automated warehousing systems proposed by Wang et al. [1]. To synchronize products and information flow, both the RFID-inventory management system and the warehousing control system need to be integrated in order to communicate effectively through a developed interface on which a framework of this on-going work is presented in this paper. The methodology for systems integration including hardware and software were identified and proposed as part of this work.

\section{REFERENCES}

[1] Q. Wang, R. McIntosh, and M. Brain, "A new-generation automated warehousing capability," International Journal of Computer Integrated Manufacturing, vol. 23, no. 6, pp. 565-573, 2010.

[2] M. K. Lim, W. Bahr, and S. C. H. Leung, "RFID in the warehouse: a literature analysis (1995-2010) of its applications, benefits, challenges and future trends," International Journal of Production Economics, vol. 145, no. 1, pp. 409-430, 2013.

[3] H. Wang, S. Chen, and Y. Xie, "An RFID-based digital warehouse management system in the tobacco industry: a case study," International Journal of Production Research, vol. 48, no. 9, pp. 2513-2548, 2010.

[4] H. K. H. Chow, K. L. Choy, W. B. Lee, and K. C. Lau, "Design of an RFID case-based resource management system for warehouse operations," Journal of Business Logistics, vol. 30, no. 4, pp. 561-576, 2006.

[5] G. Liu, W. Yu, and Y. Liu, "Resource management with RFID Technology in automatic warehouse system," in Proc. IEEE/RSJ International Conference on Intelligent Robots and Systems, 2006, pp. 3706-3711.

[6] T. C. Poon, K. L. Choy, H. K. H. Chow, H. C. W. Lau, F. T. S. Chan, and K. C. Ho, "An RFID case-based logistics resource management system for managing order-picking operations in warehouses," Expert Systems with Applications, vol. 36, no. 4, pp. 8277-8301, 2009.

[7] J. S. L. Ting and A. H. C. Tsang, "Design of an RFID-based inventory control and management system: A case study," The West Indian Journal of Engineering, vol. 34, no. 1-2, pp. 70-79, 2012.

[8] A. D. Ross, D. Twede, R. H. Clarke, and R. H. Ryan, "A framework for developing implementation strategies for a radio frequency identification (RFID) system in a distribution centre environment," Journal of Business Logistics, vol. 30, no. 1, pp. 157-183, 2009.

[9] E. Sahin and Y. Dallery, "A literature review on the impact of inventory data record inaccuracies on inventory management and the potential of the RFID technology to tackle this issue," Ecole Centrale Paris, pp. 1-16, 2007.

[10] O. E. Cakici, H. Groenevelt, and A. Seidmann, "Using RFID for the management of pharmaceutical inventory - system optimization and shrinkage control," Decision Support Systems, vol. 51, pp. 842-852, 2011.

[11] Z. Xu, X. G. Ming, J. Zhou, W. Song, L. He, and M. Li, "Management optimisation based on dynamic SKU for RFID-enabled warehouse management in the steel supply chain," International Journal of Production Research, vol. 51, no. 10, pp. 2981-2996, 2013.

[12] Y. Liu and D. Wang, "An RFID middleware business process integration framework based on EPC modeling and complex event processing," in Proc. International Conference on Computer Sciences and Convergence Information Technology, 2009, pp. 64-69.

[13] X. G. Zhou and W. Long, "The research of network architecture in warehouse management system based on RFID and WSN integration," in Proc. International Conference on Automation and Logistics, 2008, pp. 2556-2560.

[14] W. K. Jehng, S. Peng, and W. H. Huang, "Using LabVIEW to Integrate RFID system and database for supply chain efficiency improvement," International Journal of Intelligent Control and Systems, vol. 13, no. 3 , pp. 189-195, 2008.

[15] J. Yoo and Y. Park, "An intelligent middleware platform and framework for RFID reverse logistics," International Journal of Future Generation Communication and Network, vol. 1, no. 1, pp. 75-82, 2008.

[16] C. M. Liu and L. S. Chen, "Applications of RFID Technology for improving production efficiency in an integrated-circuit packaging house," International Journal of Production Research, vol. 47, no. 8 , pp. 2203-2216, 2009.

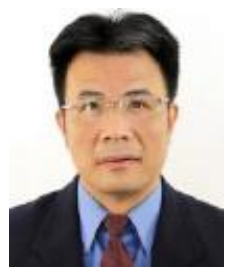

Qian Wang is a senior lecturer, School of Engineering, University of Portsmouth, UK. He has broad interests in manufacturing systems design, information systems for manufacturing, logistics and supply chain integration, sustainable manufacturing and lean production, and digital manufacturing. 


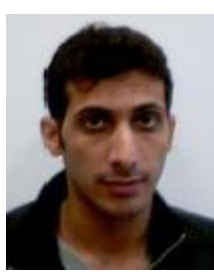

Saleh Alyahya is currently a researcher, School of Engineering, University of Portsmouth, UK. He owns a B.E in communications engineering and a M.Sc in technology management both from the University of Portsmouth, UK. His current research interests cover a field in communication networks and manufacturing systems.

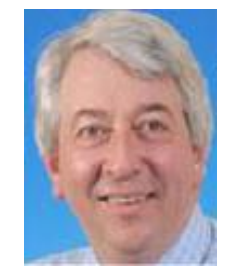

Nick Bennett is a professor, School of Engineering, University of Portsmouth, UK. He is the director of manufacturing research group with broad interests of academic and industrial research activities particularly in engineering manufacture and innovation.

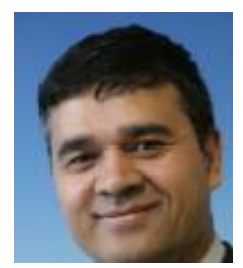

Hom Dhakal is a senior lecturer, School of Engineering, University of Portsmouth, UK. His main research areas include lean manufacturing, polymer composites and nanocomposities, sustainable hybrid composites, and bio-based materials. 